\title{
Scanning electron microscopic study of monofilament suture knots
}

\begin{abstract}
Aims/Background-Interrupted corneal sutures are used routinely by many cataract and corneal surgeons. A slim compact knot facilitates burial and allows atraumatic suture removal, with decreased risk of wound dehiscence. The size and morphology of knots tied in different configurations were investigated.

Methods-10-0 nylon suture material was used to tie knots on porcine corneal cataract sections using 2/1/1 (reef and granny), 3/1/1, and 1/1/1/1 (slip knot) configurations. 10-0 Mersilene was used to tie $2 / 1 / 1$ reef knots. Scanning electron micrographs of the knots were digitised and their maximal lengths and widths estimated. In addition, the area of each knot was calculated and used as an index of its volume.
\end{abstract}

Results-2/1/1 reef knots were significantly smaller in all dimensions compared with the other knots (all $p<0.03$ ) except for the maximal width of $2 / 1 / 1$ granny knots which were no wider than the 2/1/1 granny knots. The Mersilene knots were larger than the nylon ones.

Conclusion-The use of $2 / 1 / 1$ reef knots is recommended for corneal suturing.

(Br f Ophthalmol 1996; 80: 164-167)

Considerable ocular morbidity has been reported to be associated with monofilament nylon corneal sutures, mainly owing to the gradual biodegradation of the suture with time. ${ }^{1-3}$ Many authors recommend routine removal of all corneal nylon sutures to minimise late complications. ${ }^{4}$ Non-degradable sutures are also used, but often these have to be removed in patients with corneal sections and following penetrating keratoplasty in order to reduce astigmatism or in the event of other complications such as suture abscesses.

There are many techniques of knot tying and configurations of surgical knots in use in ophthalmic surgery, but there have been no studies relating these to knot size and shape, which may be important in facilitating knot burial and minimising corneal trauma during suture removal. The latter would in turn reduce the risk of wound dehiscence. Suture material may also be a factor influencing knot size and shape. The purpose of this study was to compare the size and shape of different knot configurations of two monofilament suture materials, in order to recommend the 'best' corneal suture method to minimise corneal trauma during knot burial and removal.
Materials and methods

Corneal sections were performed on porcine eyes by a single surgeon (CSCL), and sutured using interrupted monofilament sutures. The sutures used were 10-0 nylon (Alcon Laboratories UK, Watford) and 10-0 polyester (Mersilene; Ethicon). The suture knots were then buried into the cornea, cut and removed, and mounted in a similar orientation for scanning electron microscopy. Four different knot configurations with the nylon monofilament were assessed: a $2 / 1 / 1$ reef (square) knot, a 2/1/1 granny knot, a 3/1/1 reef knot, and a $1 / 1 / 1 / 1$ slip knot, and five examples of each type of knot were examined in order to reduce variability. Five knots were tied using the 10-0 Mersilene in a 2/1/1 reef knot configuration.

Following scanning electron microscopy, the knot micrographs were digitised on a digitising tablet by a masked observer (WYC) and estimations of their size calculated. For each knot the maximal knot width at 90 degrees to the suture orientation in the cornea, and the maximal length along the suture were measured, as well as knot area. All knots were scanned at the

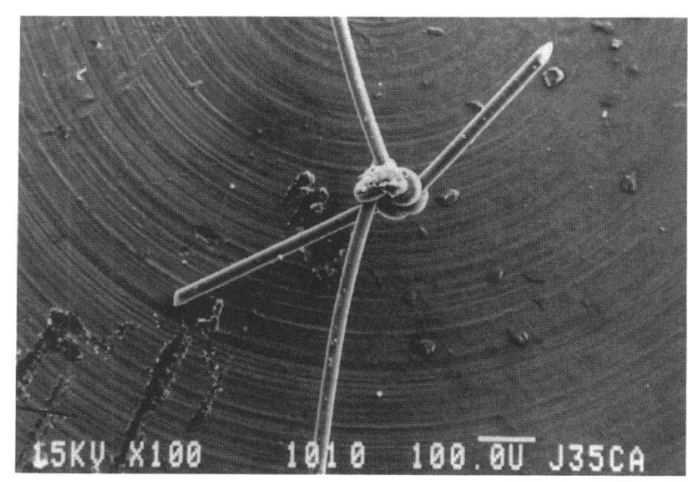
Fig $1 \mathrm{~A}$

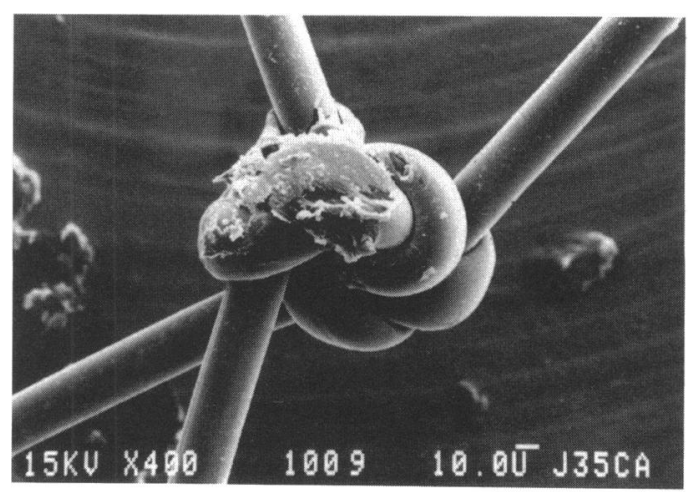

Fig $1 B$

Figure 1 Photomicrographs of an example of a 2/1/1 reef knot tied with 10-0 nylon. (A) Shows the orientation of the suture, with the long ends placed as they were in the cornea, and the short cut ends protruding. The knot (still with some epithelial debris) is shown in (B). 
same magnification, and several measurements of the suture width were taken for each knot, to confirm the absence of significant difference in magnification between the photomicrographs. The area of knot was calculated by computer using data from the digitised images. The different knot types were then compared using

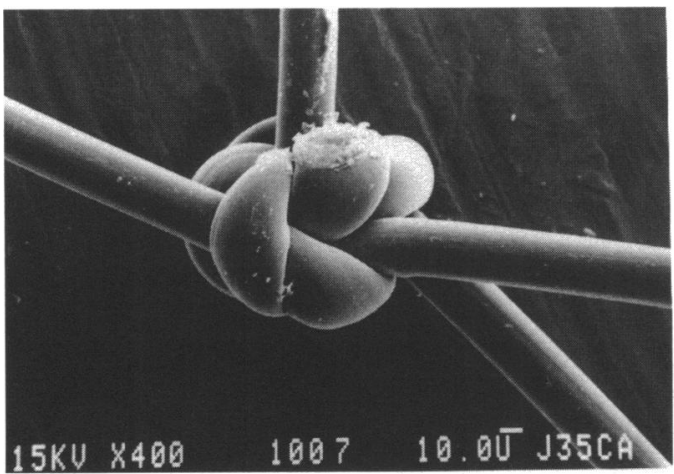

Fig $2 A$

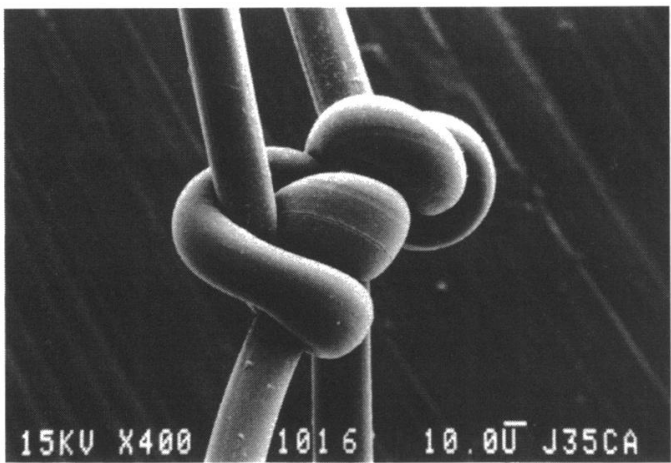

Fig $2 B$

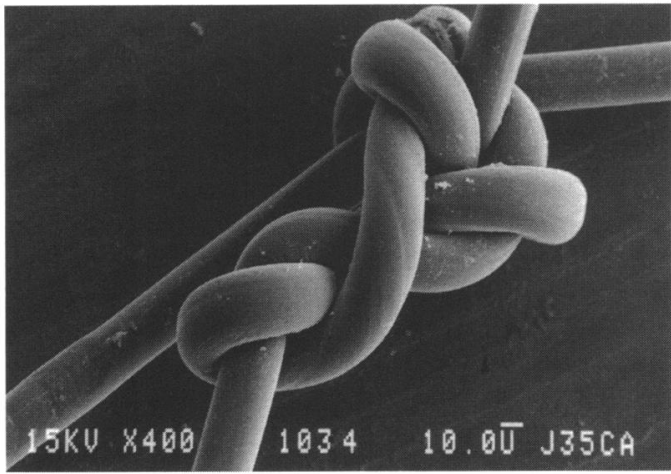

Fig $2 C$

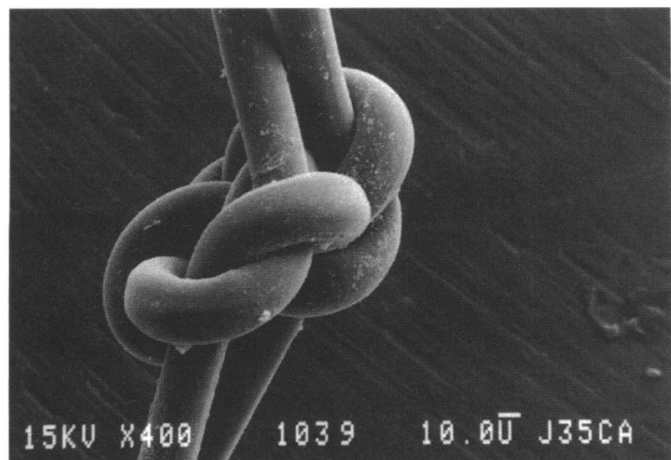

Fig $2 D$

Figure 2 Illustration of an example of each type of knot tied with 10-0 nylon. (A) Shows a 2/1/1 reef knot, $(B)$ $2 / 1 / 1$ granny knot, (C) a 3/1/1 reef knot, and (D) $a$ 1/1/1/1 slip knot.

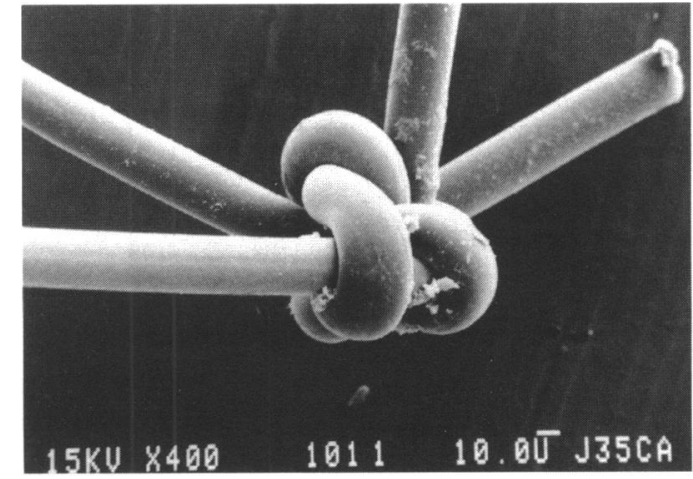

Fig $3 A$

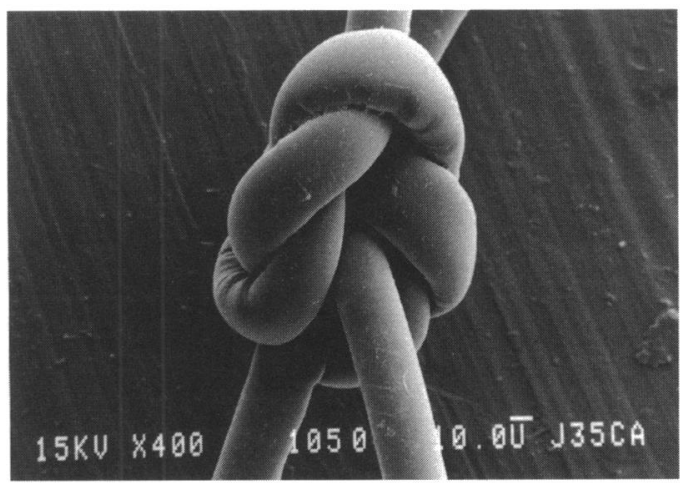

Fig $3 B$

Figure $3(A)$ Illustrates a 2/1/1 reef knot tied with 10-0 nylon, compared with (B) showing a 2/1/1 reef knot tied with 10-0 Mersilene.

non-parametric (Mann-Whitney $U$ test) and parametric (unpaired $t$ test) statistical methods.

\section{Results}

Figure 1 illustrates a photomicrograph of an example of a $2 / 1 / 1$ reef knot tied with $10-0$ nylon. Figure 1A shows the orientation of the suture, with the long ends placed as they were in the cornea, and the short cut ends protruding. The knot (still with some epithelial debris) is shown in Figure 1B. Figure 2 shows an example of each type of knot. The 2/1/1 reef knot appears more compact than the 2/1/1 granny knot, the $3 / 1 / 1$ reef knot, and the 1/1/1/1 slip knot. Figure 3B shows a $2 / 1 / 1$ reef knot tied with 10-0 Mersilene, against a similar knot tied with 10-0 nylon (Fig 3A). Again, the 10-0 Mersilene knot appears larger than the 10-0 nylon suture tied in the same knot.

The mean knot sizes are shown in Table 1. The diameter of the suture of each type of knot using 10-0 nylon was $26.0 \mu \mathrm{m}$, and the diameter of the 10-0 Mersilene was $29 \cdot 1 \mu \mathrm{m}$, representing a significant difference $(p=0 \cdot 01) .10-0$ sutures are defined as filaments of diameter between 22 and $30 \mu \mathrm{m}$. Although all dimensions of the 10-0 Mersilene knots were larger than those of comparable 10-0 nylon knots, direct comparison was not made because of the difference in suture material diameter. Comparisons between the different knot types using 10-0 nylon are shown in Table 2 . They show that in comparison with a $2 / 1 / 1$ reef knot the dimensions of length, width, and area of the $2 / 1 / 1$ granny knot, the $3 / 1 / 1$ reef knot, and the $1 / 1 / 1 / 1$ slip were significantly greater. The 
Table 1 Suture knot dimensions

\begin{tabular}{lrllllr}
\hline Knot type & $\begin{array}{l}\text { Area } \\
\left(\mu m^{2}\right)\end{array}$ & $S D$ & $\begin{array}{l}\text { Max width } \\
(\mu m)\end{array}$ & $S D$ & $\begin{array}{l}\text { Max length } \\
(\mu m)\end{array}$ & $S D$ \\
\hline Nylon: & & & & & & \\
2/1/1 Reef & 8993 & 1282 & 105 & $11 \cdot 7$ & 114 & $7 \cdot 6$ \\
2/1/1 Granny & 13509 & 3319 & 116 & $18 \cdot 1$ & 168 & $43 \cdot 0$ \\
$\begin{array}{l}\text { 3/1/1 Reef } \\
\text { 1/1/1/1 Slip }\end{array}$ & 16064 & 2507 & 132 & $18 \cdot 3$ & 191 & $53 \cdot 8$ \\
$\begin{array}{l}\text { Mersilene: } \\
\text { 2/1/1 Reef }\end{array}$ & 11415 & 1504 & 159 & $25 \cdot 6$ & 98 & $9 \cdot 7$ \\
\hline
\end{tabular}

only non-significant difference was the maximal width of the $2 / 1 / 1$ granny knot which was no wider than the $2 / 1 / 1$ reef knot.

\section{Discussion}

Our study demonstrates that corneal sutures tied as $2 / 1 / 1$ reef knots result in the smallest knot sizes compared with other methods. This configuration should result in easier knot burial and the least trauma to the cornea when removing the suture. There have been no studies observing the size of knots in ophthalmic surgery, but in the general surgical literature there are reports that foreign body material in the wound gives rise to an inflammatory response, impaired wound healing, and decreased resistance to wound infection. ${ }^{5-7}$ Not only is the knot the part of the suture representing the greatest amount of foreign material, but it also causes the highest degree of mechanical trauma of the suture loop. There is evidence that the inflammatory response and adhesion formation surrounding surgical sutures are most pronounced at the site of the knot. ${ }^{8}$ Inflammation is undesirable in corneal surgery as it may cause neovascularisation and precipitate corneal graft rejection. A study by Trimbos et al found the size of knot to be dependent on suture material, the type of knot, and suture diameter. ${ }^{5}$

Minimising the size of knot would seem to be a reasonable aim, but in corneal surgery an important consideration is the reproducibility and ease of tying knots to the desired tension to reduce astigmatism. This study shows that knots tied with 10-0 Mersilene are larger than those tied with 10-0 nylon (no doubt in part due to the larger suture diameter). There seems to be no difference in the astigmatism induced in corneal surgery between nylon and Mersilene sutures, ${ }^{9}$ but it must be remembered that Mersilene does not cause the considerable morbidity that the decay of nylon sutures engenders. ${ }^{1011}$ We found Mersilene more

Table 2 Statistical comparisons between knots tied with 10-0 nylon

\begin{tabular}{lll}
\hline Comparison & $\begin{array}{l}\text { Mann-Whitney } \\
U \text { test }(p)\end{array}$ & $\begin{array}{l}\text { Unpaired t test } \\
(p)\end{array}$ \\
\hline 2/1/1 Reef $v$ 2/1/1 granny: & & \\
Area & 0.01 & 0.02 \\
Width (max) & 0.52 & 0.28 \\
Length (max) & 0.02 & 0.04 \\
2/1/1 Reef $v$ 3/1/1 reef: & 0.01 & \\
Area & 0.03 & 0.005 \\
Width (max) & 0.06 & 0.03 \\
Length (max) & 0.04 & 0.03 \\
2/1/1 Reef $v 1 / 1 / 1 / 1$ slip: & 0.009 & 0.003 \\
Area (max) & 0.07 & 0.04 \\
Width (max) & 0 & \\
Length (max) & & \\
\hline
\end{tabular}

difficult to handle than nylon, and this factor might influence the reproducibility of knot tensions. 11-0 Mersilene may be easier to handle. ${ }^{11}$

Trimbos et al found that reef (or square) knots were smaller than slip knots, ${ }^{5}$ and we have confirmed this, as well as suggesting that 'granny' knots are also larger than reef knots. Some ophthalmic surgeons have suggested that tying a granny knot is easier and more adjustable than the standard surgeon's reef knot. ${ }^{1213}$ However, there are no comparative data between suture induced astigmatism of the different knot types, and so if the two are presumed to be comparable, then we would suggest that a reef knot is employed as the suture volume is smaller. The problem of non-control of knot tension arises from not refilling the anterior chamber and not locking the knots at the first throw. Locking at the first throw also facilitates the tying of a compact knot.

Security and strength of the knot are important, and it has been shown (with larger suture materials) that the addition of more than two throws after a starting knot offers no advantage for any sort of knot. ${ }^{14}$ Although the 'standard' general surgeon's knot (a double throw then a single, tied in a reef knot) can slip, all the knots we tested break predominantly when dynamically loaded beyond their strength, rather than slip. ${ }^{15} 16$ This suggests that they have a comparable strength, although these findings may not be applicable to monofilament suture material.

\section{Conclusion}

This study suggests that the $2 / 1 / 1$ reef knot configuration using 10-0 monofilament suture material in corneal cataract surgery creates the smallest knot size, and so should be adopted. The same would apply to interrrupted sutures in all forms of keratoplasty. Although 10-0 nylon forms a smaller knot than 10-0 Mersilene, the disadvantages of nylon biodegradation and its consequences should also be borne in mind.

1 Acheson JF, Lyons CJ. Ocular morbidity due to monofilament nylon corneal sutures. Eye 1991; 5: 106-12.

2 Confino J, Brown SI. Bacterial endophthalmitis associated with exposed monofilament sutures following corneal with exposed monofilament sutures following co

3 Balyeat HD, Davis RM, Rowsey J. Nylon suture toxicity after cataract surgery. Ophthalmology 1988; 95: 150914.

4 Jackson $H$, Bosanquet $R$. Should nylon sutures be routinely removed? Br f Ophthalmol 1991; 75: 663-4.

5 Trimbos JB, Brohim R, van Rijssel EJC. Factors relating to the volume of surgical knots. Int f Gynecol Obstet 1989; 30: 355-9.

6 Blomstedt B, Osterberrg B. Suture materials and wound infection. An experimental study. Acta Clin Scand 1978; infection.

7 Edlich RF, Panek PH, Rodeheaver GT, Turnbull VG Kurtz LD, Edgerton MT. Physical and chemical conKurtz LD, Edgerton MT. Physical and chem
figuration of sutures. Ann Surg 1973; 171: 679.

8 Luciano AA, Hauser KS, Benda J. Evaluation of commonly used adjuvants in the prevention of postoperative adhesions. Am $\mathcal{F}$ Obstet 1983; 146: 88.

9 Drews RC. Astigmatism after cataract surgery: nylon versu Mersilene. Ophthalmic Surg 1989; 15: 61-9.

10 Frueh BE, Feldman ST, Feldman RM, Sossi NP, FruchtPery J, Brown SI. Running nylon suture dissolution after penetrating keratoplasty. Am $\mathcal{f}$ Ophthalmol 1992; 113: 406-11.

11 King AJ, Deane J, Sandford-Smith J. In situ degradation of 11/0 polyester (Mersilene) suture material following cataract surgery. Eye 1994; 8: 676-9 
12 Phillips CI. Granny knot for interrupted 10-0 nylon sutures in cataract sections. Ophthalmic Surg 1993; 24: 109-12.

13 Harris DJ, Waring GO. A granny-style slip knot for use in eye surgery. Refract Corneal Surg 1992; 8: 396-8.

14 Brown RP. Knotting technique and suture materials. $B r f$ Surg 1992; 79: 399-400.
15 Brouwers JE, Oosting H, de Haas D, Klopper PJ. Dynamic loading of surgical knots. Surg Gynecol Obstet 1991; 173: 443-8.

16 Zimmer CA, Thacker JG, Powell DM, Bellian KT, Becker $\mathrm{DB}$, Rodeheaver GT, et al. Influence of knot configuration and tying technique on the mechanical performance of sutures. F Emerg Med 1991; 9: 107-13. 